\title{
TERAPIA CONDUCTUAL INTEGRATIVA DE PAREJA: DESCRIPCIÓN GENERAL DE UN MODELO CON ÉNFASIS EN LA ACEPTACIÓN EMOCIONAL
}

\section{INTEGRATIVE BEHAVIORAL COUPLE THERAPY: OVERVIEW OF A MODEL WITH EMPHASIS ON EMOTIONAL ACCEPTANCE}

\author{
Luis Francisco Vargas Madriz \\ Ronald Ramírez Henderson**
}

RESUMEN

Este artículo hace un recuento de la Terapia Conductual Integrativa de Pareja (TCIP), la cual es un modelo de intervención con énfasis en la aceptación de las diferencias entre los miembros de la pareja, permitiendo que a través de estas se logre una mayor cercanía. El artículo hace una descripción general de los fundamentos teóricos, la formulación práctica, las estrategias de intervención y las habilidades clínicas necesarias en los terapeutas. Se finaliza con un análisis crítico de la utilización de la TcIP en Costa Rica.

PALABRAS CLAVE: PSICOLOGÍA * TERAPIA * ASPECTOS PSICOLÓGICOS * SALUD MENTAL * COMPORTAMIENTO

\section{ABSTRACT}

This article reviews the Integrative Behavioral Couple Therapy (IBCT), which is a clinical intervention model that emphasizes in the acceptance of the differences between members of the couple, allowing them to grow closer in those differences. The article states the theoretical foundations of the therapy, its formulations for the session structure, its interventions strategies and the clinical skills required in the therapists. It finalizes with a critical analysis of the employment of IBCT in Costa Rica.

KEYWORDS: PSYCHOLOGY * THERAPY * PSYCHOLOGICAL ASPECTS * MENTAL HEALTH * BEHAVIOUR

\footnotetext{
* Escuela de Psicología de la Universidad de Costa Rica (UCR). luis.vargasmadriz@ucr.ac.cr

** Escuela de Psicología de la Universidad de Costa Rica (UCR). ronald.ramirez@ucr.ac.cr
} 


\section{INTRODUCCIÓN}

La Terapia Conductual Integrativa de Pareja (TCIP) es una propuesta de intervención psicoterapéutica, la cual hace hincapié en que las personas acepten patrones de comportamiento considerados como inadmisibles en sus parejas (Atkins, Eldridge, Baucom y Christensen, 2005; Barrero, 2008; Cano y Leonard, 2006; Christensen, Atkins, Baucom y Yi, 2010; Christensen y Jacobson, 2000; Cordova, Cautilli, Simon y Sabag, 2006; Jacobson y Christensen, 1996; Jacobson, Christensen, Prince, Cordova y Eldridge, 2000; South, Doss y Christensen, 2010).

La TCIP es una postura del conductismo radical (Baum, 1994; Skinner, 1969 y 1974) y se enmarca dentro de las terapias de conducta de la tercera generación debido a que retoma el contextualismo (Hayes, 1987). Empero, la aproximación práctica está intrínsecamente marcada por la Terapia de Pareja Conductual Tradicional (ТРСТ) (Barrero, 2008; Baucom, Sevier, Eldridge, Doss y Christensen, 2011; Cordova et ál., 2006; Cordova, Jacobson y Christensen, 1998; Doss, Meng, Sevier, Atkins y Christensen, 2005; Jacobson y Christensen, 1996; Jacobson et ál., 2000; Jacobson y Margolin, 1979).

El componente de aceptación emocional dentro de la TCIP no implica la concepción de patrones de resignación ante las conductas de la pareja, sino más bien una forma de aprender a vislumbrar las dificultades de la pareja como un medio a través del cual se puede encontrar una mayor cercanía e intimidad con la persona (Atkins, Eldridge et ál.,2005; Barrero, 2008; Christensen y Jacobson, 2000; Doss et ál., 2005; Jacobson y Christensen, 1996; Jacobson et ál., 2000; Lebow, Chambers, Christensen y Johnson, 2012).

La aceptación de diferencias irreconciliables se convierte en un elemento de cohesión para los miembros de la pareja, más que uno de conformismo para una de las personas involucradas, por lo que la aceptación de la conducta problemática se convierte en un elemento reforzante de la relación. Es importante señalar que la aceptación va a estar conformada por dos componentes fundamentales; el primero se relaciona con lo señalado anteriormente, en el que se trata de convertir las problemáticas en medios para alcanzar la intimidad entre los miembros de la pareja (Barrero, 2008; Doss et ál., 2005; Jacobson y Christensen, 1996). El segundo componente fundamental de la aceptación dentro de la TCIP, es que los consultantes deben de desistir en sus intentos por cambiar el comportamiento de la otra persona. El terapeuta tiene la función de ayudar a los sujetos a adquirir una visión más completa del comportamiento de su pareja, renunciando a la idea que las diferencias que existen entre ambos no son tolerables y que por consiguiente, la única solución sería el cambio (Barrero, 2008; Christensen y Jacobson, 2000; Jacobson y Christensen, 1996; Jacobson et ál., 2000).

La TCIP es una propuesta que brinda una visión integral de la conducta de las personas dentro de la pareja, partiendo del enfoque contextual (Epstein y Baucom, 2002; Hayes, 1987), en el que se hace un análisis funcional de las variables controladoras que se encuentran determinando la conducta problemática, para que a partir de manipulaciones en las condiciones antecedentes o en las condiciones consecuentes al comportamiento o en su defecto la conducta misma, se pueda alcanzar el bienestar dentro de la relación de pareja (Jacobson y Christensen, 1996).

\section{TERAPIA CONDUCTUAL INTEGRATIVA DE PAREJA}

Al ser la TCIP un modelo contextual, se hace crítico tomar en cuenta los "determinantes ambientales del comportamiento tanto presentes como históricos”, así como, las "variables personales de los miembros de la pareja" (Jacobson y Christensen, 1996; Jacobson et ál., 2000).

Los "determinantes ambientales del comportamiento" hacen referencia a los factores que se encuentran presentes en el medio en que se desarrollan los miembros de la pareja $y$ que pueden relacionarse con la situación problemática (Epstein y Baucom, 2002; Jacobson y Christensen, 1996; Jacobson y Margolin, 1979). En esta categoría se toman en consideración no solo los estímulos antecedentes que se encuentran antes de la emisión de una determinada conducta, sino que también se presta atención 
a las consecuencias que suceden contingentes al comportamiento de alguno de los miembros de la pareja.

La aproximación es relevante debido a las particularidades contextuales que la conducta problemática pueda traer consigo, por lo que una aproximación que deje de lado la realización de un análisis funcional del comportamiento, va a carecer de resultados eficaces a largo plazo. Empero, la propuesta de la TCIP no solo toma en consideración este tipo de elementos, sino que también considera los factores individuales que pueden estar afectando, tanto a nivel individual como a la pareja misma.

El análisis de la situación problema estaría incompleto sin la inclusión de las "variables personales de los miembros de la pareja", que sin duda alguna tienen influencia en la dinámica de la relación entre ambos. Es trascendente considerar elementos relacionados con las "atribuciones" que hacen las personas en torno al comportamiento de sus parejas, las "expectativas" que mantienen en torno a las relaciones sentimentales, así como, los "estándares" manejados como requisitos necesarios en la persona que se elija como la pareja (Epstein y Baucom, 2002; Jacobson y Christensen, 1996; Jacobson y Margolin, 1979).

Es primordial que el análisis funcional en estos distintos escenarios considere los tres niveles de respuesta, recordando que todo comportamiento tiene correlatos "cognitivos, conductuales y emocionales", a los que se les debe prestar atención para la adecuada comprensión de la situación que se encuentre afectando a los miembros de la pareja en la actualidad (Epstein y Baucom, 2002; Jacobson y Christensen, 1996).

La TCIP hace un esfuerzo por enmarcarse dentro del contexto (presente e histórico) en que suceden las conductas de los miembros de la pareja, además cuenta con dos componentes principales para hacerle frente a estas: el intercambio de conductas positivas y el entrenamiento en comunicación y en solución de problemas (Barrero, 2008; Jacobson y Margolin, 1979). Estos dos procedimientos de intervención son tomados de la ТРСТ, en donde se demostró que eran sumamente importantes (Jacobson y Margolin, 1979).
El "intercambio de conducta" se centra principalmente en fomentar los intercambios positivos que mantienen las personas dentro de las relaciones de pareja, a la vez que se disminuyen la cantidad de intercambios negativos (Barrero, 2008; Jacobson y Margolin, 1979). El propósito detrás de esta aproximación es que las personas puedan contar con una mayor cantidad de estímulos reforzantes que los haga sentirse satisfechos con la otra persona y reducir las interacciones negativas, las cuales son las que ponen un mayor énfasis en las problemáticas que se encuentran viviendo como pareja. Se busca de esta manera que se desarrolle y se mantenga en forma consistente, un énfasis en la reciprocidad positiva en la interacción de la pareja (Jacobson y Margolin, 1979).

Barrero (2008) menciona que este modelo de intervención es definido en sí mismo como instigador, debido a que incita a los miembros de la pareja a aumentar la frecuencia de comportamientos reforzantes, a la vez que se disminuyen los aversivos. Asimismo, la propuesta de la TPCT cuenta con el elemento de entrenamiento en comunicación y en solución de problemas. La base de dicha propuesta se sustenta en que la mayoría de las problemáticas presentadas dentro del contexto de pareja van a mostrar en sus orígenes un déficit en las habilidades de comunicación o en las habilidades para la resolución de conflictos, por lo que se muestra como indispensable el desarrollo de este tipo de estrategias.

La TPCT hace también énfasis en el concepto de "reciprocidad", en dónde es imposible vislumbrar el vínculo de pareja sin considerar la interdependencia existente entre ambos individuos, por lo que el comportamiento de cualquiera de las personas va a estar influenciado por el comportamiento de la otra persona, de forma que existirá una tendencia a responder en la misma frecuencia e intensidad de la conducta original en un cierto periodo de tiempo (Jacobson y Margolin, 1979).

Por otro lado, la TPCT cuenta con una serie de dificultades que han minado su utilización dentro del contexto clínico. Por ejemplo, esta propuesta hace hincapié en el compromiso, la acomodación y la colaboración que debe 
tenerse dentro del proceso de intervención, por lo que aquellas parejas que no cumplían con dichos criterios no mostraban buenos resultados una vez finalizada la intervención; en parte debido a que la TPCT no los ayudaba a resolver realmente sus problemas y además, porque los cambios gestados dentro del contexto terapéutico se estaban logrando por comportamiento gobernado por reglas y en particular, el seguimiento de instrucciones dadas por el contexto terapéutico (Jacobson y Christensen, 1996; Skinner, 1969 y 1974), que más adelante no era posible mantener cuando la pareja se exponía a las contingencias naturales (Atkins, Berns et ál., 2005; Barrero, 2008; Baucom et ál., 2009; Christensen et ál., 2010; Christensen et ál., 2004; Christensen et ál., 2006; Jacobson y Christensen, 1996).

Asimismo, la ТРСт mostraba excelentes resultados con aquellas parejas que ingresaban a terapia con el compromiso de cambio, mientras que las personas que no contaban con este incentivo generalmente no conseguían una mejora con la intervención (Atkins, Berns et ál., 2005; Barrero, 2008; Baucom et ál., 2009; Christensen et ál., 2010; Christensen et ál., 2004; Christensen et ál., 2006; Jacobson y Christensen, 1996). Con cierta frecuencia, aún haciendo esfuerzos terapéuticos por desarrollar el set de colaboración propuesto por Jacobson y Margolin (1979), no se lograba desarrollar o mantener, y ello incidía en el nivel de compromiso de la pareja.

Entonces, el principal elemento que es necesario incorporar a la propuesta de intervención es la "aceptación". La TCIP retoma las premisas teóricas y prácticas de la ТРCT e incluye este componente que se considera necesario para completar la propuesta de intervención. La TCIP parte del supuesto que pueden existir patrones de comportamiento incompatibles entre los miembros de las parejas que no pueden modificarse, por lo que la aceptación emocional se vislumbra como el objetivo terapéutico principal que trata de alcanzarse con este tipo de intervención (Atkins, Berns et ál., 2005; Barrero, 2008; Baucom et ál., 2009; Cordova et ál., 1998; Christensen et ál., 2010; Christensen et ál., 2004; Christensen et ál.,
2006; Jacobson y Christensen, 1996; Jacobson et ál., 2000).

La TCIP trata de sacar ventaja de las diferencias entre las personas miembros de la relación de pareja, de las dificultades que se encuentran experimentando, así como de las problemáticas que se consideran irresolubles para generar cercanía, pues como se había señalado: "(...) las incompatibilidades significan que uno o ambos miembros de la pareja están deprivados de refuerzo, y están expuestos a una estimulación aversiva" (Christensen y Jacobson, 1996 citados en Barrero, 2008: 100).

Los planteamientos de intercambio positivos de conducta y el desarrollo de habilidades de comunicación y de resolución de conflictos, en conjunto con las premisas de aceptación emocional dentro de la TCIP, hacen que dentro de la dinámica de pareja se generen estados de mayor cercanía, mayor tolerancia y consecuentemente, una mayor posibilidad hacia el cambio (Barrero, 2008; Christensen et ál., 2010; Doss et ál., 2005; Jacobson y Christensen, 1996; Jacobson et ál., 2000). Aunque el objetivo principal no esté centrado en la modificación del comportamiento, la conjugación de elementos hace que la modificación de la conducta problemática llegue a ser una posibilidad muy viable, por lo que se alcanza un nivel de mejoría que refiere directamente al motivo de consulta inicial.

\section{PROCESO TERAPÉUTICO Y ESTRUCTURA DE LAS SESIONES DE INTERVENCIÓN}

El proceso terapéutico se inicia con la fase de evaluación conductual, la cual se desarrolla en cuatro sesiones: la primera sesión se lleva a cabo con ambos miembros de la pareja, la segunda y la tercera sesión se realizan de manera individual con cada cónyuge, $y$ la cuarta sesión se lleva a cabo de manera conjunta para brindar la retroalimentación (Jacobson y Christensen, 1996).

Los objetivos de esta evaluación son "generar la formulación con su respectivo plan de intervención, brindar la retroalimentación a la pareja, y producir un impacto terapéutico en la pareja”. Para llevar a cabo este proceso de evaluación, el terapeuta de TCIP recurre a la entrevista clínica conductual y 
a la aplicación de algunos instrumentos de evaluación tales como: la "Escala de Ajuste Diádica", el "Inventario de Satisfacción Marital", el "Inventario del Estatus Marital", el "Cuestionario de Áreas de Cambio", entre otros (Jacobson y Christensen, 1996).

Inmediatamente después de la sesión de retroalimentación, se procede a desarrollar el plan de intervención propuesto $y$ aceptado por la pareja. En esta fase se realizan sesiones en donde intervienen ambos cónyuges en forma conjunta. Sin embargo, al igual que en la TPCT, existe la opción de poder intervenir a nivel individual si las condiciones lo requieren (Epstein y Baucom, 2002; Jacobson y Christensen, 1996; Jacobson y Margolin, 1979).

Una vez alcanzados los objetivos terapéuticos se procede al cierre de la intervención. Jacobson y Christensen (1996) recomiendan llevar a cabo una sesión de recapitulación, en donde los consultantes evalúan el proceso que se ha seguido. Hacia el final del proceso es recomendable ir desvaneciendo sustractivamente la presencia del terapeuta y por ello las sesiones se van distanciando entre ellas. También es recomendable el realizar sesiones de fortalecimiento o booster sessions (Jacobson y Christensen, 1996).

Tal y como se señaló anteriormente, la TCIP tiene como punto de partida una formulación, la cual está compuesta por tres componentes principales: el "tema", el "proceso de polarización" y la "trampa mutua" (Barrero, 2008; Jacobson y Christensen, 1996; Jacobson et ál., 2000), los cuales se describen a continuación:

$\diamond \quad$ El tema: es la descripción en palabras detalladas de la problemática principal a la que se está enfrentando la pareja en la actualidad. El objetivo principal del tema es lograr identificar la función que el comportamiento de cada uno de los miembros de la pareja tiene sobre el conflicto que se está presentando. Asimismo, este elemento permitirá obtener una visión más confiable de aquellas conductas que una vez modificadas, puedan tener impacto en otras áreas de la relación y que por consiguiente, logren mantenerse por medio de contingencias ambientales (Barrero, 2008; Christensen y Jacobson, 2000; Jacobson y Christensen, 1996; Jacobson et ál., 2000).

$\diamond \quad$ El proceso de polarización: es la descripción de las interacciones aversivas o destructivas que se han venido presentando en la relación de pareja, pudiéndose generalmente observar en este que las personas hacen esfuerzos insistentes por cambiar el comportamiento de su pareja, en intentos para que la otra persona acepte su postura frente a la dificultad. El proceso de polarización deviene justamente de dichos intentos por imponer un ajuste de la conducta en la otra persona, ya que la presencia de diferencias e incompatibilidades en una relación de pareja van a verse acrecentadas por los esfuerzos incesantes de cambio de uno de los miembros, los cuales son antagónicos a los esfuerzos del otro miembro por mantener los mismos patrones de conducta $y$ viceversa (Barrero, 2008; Christensen y Jacobson, 2000; Jacobson y Christensen, 1996; Jacobson et ál., 2000).

$\diamond \quad$ La trampa mutua: es el resultado del proceso de polarización, el cual generalmente puede percibirse como un estado de estancamiento que le ha impedido a la pareja salir del círculo vicioso de interacciones negativas que los ha estado perturbando. Ese sentimiento de estancamiento puede ser aliviado con una simple discusión que haga referencia directa a la temática, pues le permitirá a la persona sentirse comprendida y saber que su pareja le está comprendiendo gracias a la articulación brindada por la guía del terapeuta (Barrero, 2008; Christensen y Jacobson, 2000; Jacobson y Christensen, 1996; Jacobson et ál., 2000).

Desde esta perspectiva, la formulación representa: a) un contexto para poder comprender la confusión, la desesperación, la desesperanza y el dolor de la relación de pareja 
$y$ b) un vocabulario que los miembros de la pareja pueden utilizar en contra del conflicto $y$ que a su vez, les permita tomar distancia emocional para que lo puedan experimentar o vivenciar conjuntamente como una unidad (Jacobson y Christensen, 1996).

El trabajo del terapeuta de TCIP está orientado principalmente a la generación de empatía y de aceptación por parte de ambos miembros de la pareja para con el comportamiento de la otra persona, principalmente a través de la comprensión del sentimiento de estancamiento que se ha logrado comprender a través del proceso de intervención y que no solo se encuentra presente en uno de los miembros de la pareja, sino que está presente en ambos (Barrero, 2008).

Un adecuado entendimiento de estos componentes constituye la piedra angular para la formulación exitosa del consiguiente proceso de intervención. Es importante señalar que dentro de la TCIP, el proceso a seguir viene dado por las particularidades de cada una de las parejas, por lo que se hace énfasis en la adecuada evaluación de las circunstancias presentes e históricas de la pareja para tener una comprensión de la problemática, la cual permita hacer uso de las herramientas terapéuticas que se crean más convenientes.

\section{ESTRATEGIAS DE INTERVENCIÓN}

La TCIP pone el énfasis en dos focos principales de intervención: las estrategias centradas en la aceptación y las estrategias centradas en el cambio. El terapeuta tiene la responsabilidad de decidir en cada momento de la terapia si es más conveniente centrarse en un tipo de estrategias u otro. A partir de su criterio profesional, seleccionará aquellas que considere más pertinentes para la pareja (Barrero, 2008; Jacobson y Christensen, 1996).

\section{ESTRATEGIAS DE ACEPTACIÓN}

$\diamond \quad$ El uso de la aceptación para convertir los problemas en vehículos para la intimidad: en la mayoría de las ocasiones los miembros de la pareja ingresan a terapia con el objetivo de modificar de manera exclusiva los comportamientos que se encuentran manteniendo la problemática. Sin embargo, este tipo de cambios es muy difícil de alcanzar y en algunas ocasiones casi que imposible, debido a que los mismos se sustentan en diferencias fundamentales entre las personas (Barrero, 2008; Christensen y Jacobson, 2000; Jacobson y Christensen, 1996).

El objetivo de esta estrategia es permitir que las personas conciban sus diferencias en una forma más constructiva, siendo ideal que no solo se vayan de las sesiones aceptándolas, sino también utilizándolas para desarrollar una relación más cercana e íntima $y$ aunque en algunas ocasiones esto no pueda conseguirse, cuando estas diferencias se convierten en fortalezas, la necesidad de las personas por cambiar a la pareja desaparece e incluso, los miembros de la pareja aprenden a apreciarse entre sí gracias a sus diferencias y no solo por sus compatibilidades (Barrero, 2008; Christensen y Jacobson, 2000; Jacobson y Christensen, 1996).

La aceptación se logra por medio de dos procedimientos: el acercamiento empático hacia el problema y la separación unificada. En el primero, se pone en evidencia la máxima: dolor más acusación es igual al conflicto marital $y$ por el contrario, dolor sin acusación es igual a aceptación (Christensen y Jacobson, 2000; Jacobson y Christensen, 1996; Jacobson et ál., 2000). Adicionalmente, se refuerza e instiga la utilización del lenguaje basado en la experiencia propia de cada cónyuge y no en función de la experiencia del otro. Se hace énfasis en las expresiones de "revelaciones suaves" (aquellas que implican una visión vulnerable de la persona, por ejemplo: expresión de sentimientos de sufrimiento, temor o desilusión), en contraposición a las "revelaciones fuertes" (aquellas que presentan a la persona como impactante $y$ fuerte, por 
ejemplo: expresiones emocionales asociadas a la cólera y al resentimiento). La separación unificada implica describir en forma detallada la secuencia o contexto relacionado con un conflicto relevante para la pareja. Esto ayuda a realizar un análisis de carácter intelectual o racional del problema y por ello facilita el control de las implicaciones emotivas asociadas (Christensen y Jacobson, 2000; Jacobson y Christensen, 1996).

$\diamond \quad$ La tolerancia: esta estrategia tiene como principal objetivo neutralizar el impacto del comportamiento negativo de la pare$\mathrm{ja}$, de forma que los efectos sean menos adversos de lo que usualmente lo han sido. Es importante resaltar que aunque las intervenciones centradas en la tolerancia sean satisfactorias, las personas afectadas generalmente van a seguir prefiriendo que el conflicto no existiera, que el comportamiento negativo no ocurriera con tanta frecuencia o que la conducta positiva tuviera una frecuencia mayor (Barrero, 2008; Jacobson y Christensen, 1996).

La tolerancia se desarrolla por medio de los procedimientos de exposición y de moldeamiento conductual. Algunas de las estrategias utilizadas son el describir características positivas relacionadas con las conductas negativas, el practicar y simular conductas negativas entre las sesiones, además de fomentar las conductas de auto-cuidado, entre otras (Christensen y Jacobson, 2000; Jacobson y Christensen, 1996).

Jacobson y Christensen (1996) explican que la diferencia fundamental entre ambas aproximaciones es que en la primera, los conflictos van a ayudar a la pareja a desarrollar un vínculo más cercano, mientras que en la segunda, los conflictos van a seguir siendo indeseables, pero van a soportarse con mayor facilidad gracias a la intervención terapéutica. Igualmente, es importante tomar en consideración que durante las sesiones de intervención, el terapeuta va a ser quien decida qué estrategia de intervención emplear, siendo usual que se recurra a ambos tipos de manera frecuente.

\section{ESTRATEGIAS DE CAMBIO}

Las estrategias de cambio se abordaron con anterioridad, siendo las principales el "intercambio positivo de conducta" y el entrenamiento en "habilidades de comunicación y en solución de problemas".

Jacobson y Christensen (1996) indican que el empleo de estos procedimientos en servicio de una meta en conjunto, hace que la aproximación se conciba como integrativa, debido a que no importa el objetivo que cada técnica quiera conseguir en sí misma, pues las intervenciones más eficientes van a ser aquellas que sirvan tanto para la aceptación como para el cambio. La aceptación puede dirigir a un cambio y el cambio puede dirigir a la aceptación, pero lo que la TCIP realmente busca es la expresión de las problemáticas en una atmósfera de aprobación y en donde no sea necesaria la utilización de estrategias defensivas por parte de las personas involucradas (Barrero, 2008; Christensen y Jacobson, 2000; Jacobson y Christensen, 1996).

\section{HABILIDADES CLÍNICAS EN LA TERAPIA CONDUCTUAL INTEGRATIVA DE PAREJA}

Las particularidades de la TCIP hacen que sea necesario un perfil básico del terapeuta. De esta forma, los autores proponen una serie de habilidades clínicas como requisito para la adecuada puesta en marcha del modelo de intervención:

$\diamond \quad$ Atención a material relacionado al análisis funcional: es imprescindible que el terapeuta de TCIP tenga buenas habilidades de escucha, debido a que se tiene que estar atento al relato de los consultantes para poder hacer formulaciones y reformulaciones de los elementos relacionados con el análisis funcional (Jacobson y Christensen, 1996).

Es también imprescindible habilidades de observación, debido a que la información relevante no va a provenir solamente del discurso de la pareja, sino también de elementos relacionados con componentes no verbales $y$ componentes vocales 
o paralingüísticos, los cuales brindan una amplia perspectiva del comportamiento de los consultantes (Jacobson y Christensen, 1996).

Hay que recordar que el análisis funcional es una impresión secundaria obtenida a través de observaciones y del relato de los consultantes, por lo que entre más perfeccionada se encuentre esta habilidad, más probabilidades de éxito tendrá la intervención terapéutica. Conviene destacar que esta habilidad se desarrolla por medio de un control mixto entre la conducta gobernada por reglas y la conducta gobernada por contingencias (Baum, 1994; Skinner, 1969 y 1974). La primera, debido a las auto-instrucciones que el terapeuta utiliza para facilitar la adherencia del método, por lo que requiere un esfuerzo inicial intencional $y$ por consiguiente, consciente de lo que se hace y por qué se hace. Así, al actuar de acuerdo con la regla, el comportamiento del terapeuta va siendo moldeado sistemáticamente por las contingencias naturales a las cuales se expone $y$ en la medida que su capacidad de observación facilita la elaboración del análisis funcional de la conducta de la pareja, este pasa a ser más espóntaneo y fluido, al punto de convertirse en un hábito. En este punto, se dice que el comportamiento que conlleva el análisis funcional de la conducta es natural y por ende, no requiere de un control voluntario e intencional por parte del terapeuta, lo cual es el ideal.

Sensibilidad al contexto: es necesario que el terapeuta tenga la flexibilidad para dejar a un lado la agenda planificada para la sesión con base a las particularidades encontradas en el transcurso de la misma, debido a que las características que pueden presentarse en un determinado contexto van a ser más importantes que adherirse a un plan de intervención preestablecido (Christensen y Jacobson, 2000; Jacobson y Christensen, 1996).
El fijarse al plan de la agenda sin tomar en consideración los elementos surgidos dentro de la sesión, significa ignorar oportunidades cruciales que pueden marcar la diferencia en la dirección del tratamiento y en el éxito del mismo, por lo que se enmarca como una habilidad necesaria en los terapeutas de TCIP.

$\diamond \quad$ Aceptación: es indispensable que el terapeuta no mantenga una agenda oculta hacia los consultantes, en donde se trate de imponer sus propias creencias en torno al comportamiento de cada uno de los miembros de la pareja. El enfoque parte de la aceptación incondicional de la conducta de las personas, a pesar de lo aversiva que esta pueda parecerle (Jacobson y Christensen, 1996).

La TCIP se enmarca dentro de la perspectiva de que las personas cometen errores en sus relaciones de pareja debido a que se encuentran experimentando malestar, por lo que la mayoría de las acciones inadecuadas se cometen de forma automática, es decir, no deliberada. La aceptación no debe ser una petición solamente hacia la pareja, sino que el terapeuta debe iniciar con esta formulación para que la pareja a partir de esta, pueda empezar a aceptarse a sí misma con sus particularidades (Christensen y Jacobson, 2000; Jacobson y Christensen, 1996). En este sentido, se hace crítico que el terapeuta no reaccione negativamente (acusando o culpando a alguno de los cónyuges) ante las conductas provocativas por parte de los consultantes.

$\diamond \quad$ Habilidad para descubrir y mantener el foco en la formulación: es importante que el terapeuta pueda centrarse en la formulación de la TCIP, debido a la relevancia terapéutica que tiene dentro de las sesiones de intervención, así como, para el entendimiento de las problemáticas de pareja. Empero, esta habilidad requiere un gran esfuerzo por parte del terapeuta para hacer inferencias adecuadas que 
le permitan a los consultantes aprender acerca del "tema", del "proceso de polarización" y de la "trampa mutua" (Jacobson y Christensen, 1996).

$\diamond \quad$ Habilidad para mantener una atmósfera terapéutica sin confrontación pese a la presencia de conflictos severos: es necesario que el terapeuta pueda ser capaz de interrumpir las interacciones negativas sin contribuir a las acusaciones que se están realizando por uno de los miembros de la pareja o sin acrecentar el sentimiento de culpa que pueda estarse presentando en determinado contexto (Jacobson y Christensen, 1996).

Es importante que el terapeuta no tome partido, se mantenga neutral, conserve la calma, ejerza una influencia tranquilizadora, así como, no dejarse influenciar por la culpa y las acusaciones que se le están haciendo a algún miembro de la pareja o a ambos (Jacobson y Christensen, 1996).

$\diamond \quad$ Habilidad en el uso del lenguaje de forma que se adecue a las particularidades de los consultantes: es imprescindible que el terapeuta sea capaz de dar ejemplos que se muestren significativos para los miembros de la pareja, así como, utilizar las metáforas adecuadas que puedan causar un impacto en la vida de los individuos y en la problemática que se quiere abordar, por lo que se requiere que se preste atención a la utilización que hacen los consultantes del lenguaje, con el fin de poder modular las expresiones de forma que se adecuen a sus particularidades (Jacobson y Christensen, 1996).

Es crítico que en todo momento se recuerde que el enfoque de la TCIP es ideográfico, por lo que las particularidades de la intervención, así como, las habilidades necesarias en el terapeuta, están construidas de forma que se respeten las particularidades de cada uno de los individuos en su contexto socio-histórico.

\section{DISCUSIÓN: TERAPIA CONDUCTUAL INTEGRATIVA DE PAREJA EN EL CONTEXTO COSTARRICENSE}

La práctica de la TCIP es totalmente viable en nuestro país. En principio no existen limitaciones más allá de la preparación de los terapeutas que se requiere desde el punto de vista ético-profesional. Desde el punto de vista curricular, es recomendable que el profesional que desee profundizar en esta alternativa terapéutica sea capaz de comprender las bases epistemológicas del Conductismo Radical (Baum, 1994; Skinner, 1969 y 1974).

Adicionalmente, es indispensable que se haya tenido entrenamiento en Modificación de Conducta (Cognitivo-Conductual-Emocional), Evaluación Conductual, Entrevista Clínica Conductual, Intervención Conductual de Pareja e Intervención Conductual de Familia. En estas últimas áreas, se requiere necesariamente de entrenamiento en el modelo TPCT (Jacobson y Margolin, 1979) y por supuesto, en la medida de lo posible, la certificación en el manejo de la TCIP.

Este último requisito nos remite a la necesidad de estructurar espacios de capacitación que permitan la convocatoria de profesionales extranjeros expertos en este modelo, los cuales estén en la disposición de venir a nuestro país a compartir sus conocimientos, asî como a los profesionales costarricenses responsables en su práctica profesional. Es necesario hacer énfasis en que, como profesionales de la Salud Mental, tenemos la obligación de actualizar nuestras herramientas de intervención con instrumentos que demuestren brindar resultados más eficientes y más eficaces para las personas consultantes, siendo la TCIP una alternativa viable para los practicantes en Psicología Clínica.

La propuesta no tiene requerimientos mínimos en términos de duración, por lo que el proceso de intervención psicoterapéutica se construye enteramente alrededor de las particularidades de la pareja. Asimismo, los recursos necesarios para la aplicación de la TCIP son los mismos que se requieren para la puesta en marcha de cualquier otro tipo de intervención terapéutica, por lo que no existen particularidades para este modelo. 
Finalmente, es importante resaltar que dentro de la práctica profesional de la Psicología en Costa Rica, se deben abrir espacios que permitan la incorporación de este tipo de alternativas clínicas que han demostrado su efectividad. Los programas curriculares deben ir integrando de manera progresiva estas opciones para garantizar a las personas un servicio de calidad en atención a las problemáticas que nos están siendo planteadas.

\section{REFERENCIAS BIBLIOGRÁFICAS}

Atkins, D.; Berns, S.; George, W.; Doss, B.; Gattis, K. y Christensen, A. "Prediction of response to treatment in a randomized clinical trial of Marital Therapy". Journal of Consulting and Clinical Psychology 73 (5). 2005: 893-903.

Atkins, D.; Eldridge, K.; Baucom, D. y Christensen, A. "Infidelity and Behavioral Couple Therapy: optimism in the face of betrayal". Journal of Consulting and Clinical Psychology 73 (1). 2005: 144-150.

Barrero, J. "La Terapia Marital Integrativa: una propuesta para la intervención del conflicto marital". Revista Digital de Psicología 3 (4). 2008: 95-111.

Baucom, B.; Atkins, D.; Simpson, L. y Christensen, A. "Prediction of response to treatment in a randomized clinical trial of Couple Therapy: a 2 year follow up". Journal of Consulting and Clinical Psychology 77 (1). 2009: 160-173.

Baucom, K.; Sevier, M.; Eldridge, K.; Doss, B. y Christensen, A. "Observed communication in couples two years after Integrative and Traditional Behavioral Couple Therapy: outcome and link with five year follow up". Journal of Consulting and Clinical Psychology 79 (5). 2011: 565-576.

Baum, W. Understanding behaviorism. Science, behavior and culture. New York: Harper Collins College Publishers, 1994.

Cano, A. y Leonard, M. "Integrative Behavioral Couple Therapy for chronic pain: promoting behavior change and emotional acceptance". Journal of
Clinical Psychology: In Session 62 (11). 2006: 1409-1418.

Cordova, J.; Cautilli, J.; Simon, C. y Sabag, R. "Behavior analysis of forgiveness in Couples Therapy". International Journal of Behavioral and Consultation Therapy 2 (2). 2006: 192-214.

Cordova, J.; Jacobson, N. y Christensen, A. "Acceptance versus change interventions in Behavioral Couple Therapy: impact on couples' in-session communication". Journal of Marital and Family Therapy 24 (4). 1998: 437-455.

Christensen, A.; Atkins, D.; Baucom, B. y Yi, J. "Marital status and satisfaction five years following a randomized clinical trial comparing Traditional versus Integrative Behavioral Couple Therapy. Journal of Consulting and Clinical Psychology 78 (2). 2010: 225-235.

Christensen, A.; Atkins, D.; Berns, S.; Wheeler, J.; Baucom, D. y Simpson, L. "Traditional versus Integrative Behavioral Couple Therapy for significantly and chronically distressed married couples. Journal of Consulting and Clinical Psychology 72 (2). 2004: 176-191.

Christensen, A.; Atkins, D.; Yi, J.; Baucom, D. $y$ George, W. "Couple and individual adjustment for 2 years following a randomized clinical trial comparing Traditional versus Integrative Behavioral Couple Therapy". Journal of Consulting and Clinical Psychology 74 (6). 2006: 1180-1191.

Christensen, A. y Jacobson, N. Reconcilable differences. New York: The Guilford Press, 2000.

Doss, B.; Meng, Y.; Sevier, M.; Atkins, D. y Christensen, A. "Improving relationships: mechanisms of change in Couple Therapy". Journal of Consulting and Clinical Psychology 73 (4). 2005: 624-633.

Epstein, N. y Baucom, D. Enhanced CognitiveBehavioral Therapy for couples: a contextual approach. Washington DC: American Psychological Association, 2002. 
Hayes, S. "A contextual approach to therapeutic change". Psychotherapists in clinical practice: cognitive and behavioral perspectives. Jacobson, N. (ed.). New York: The Guilford Press, 1987.

Jacobson, N. y Christensen, A. Acceptance and change in Couple Therapy: a therapist's guide to transforming relationships. New York: W.W. Norton \& Company, 1996.

Jacobson, N.; Christensen, A.; Prince, S.; Cordova, J. y Eldridge, K. "Integrative Behavioral Couple Therapy: an acceptance-based, promising new treatment for couple discord". Journal of Consulting and Clinical Psychology 68 (2). 2000: 351-355.

Jacobson, N. y Margolin, G. Marital Therapy: strategies based on social learning and behavior exchange principles. New York: Brunner/Mazel Publishers, 1979.
Lebow, J.; Chambers, A.; Christensen, A. y Johnson, S. "Research on the treatment of Couple Distress". Journal of Marital and Family Therapy 38 (1). 2012: 145168.

Skinner, B. Contingencies of reinforcement. A theoretical analysis. New York: AppletonCentury-Crofts, 1969.

Skinner, B. About behaviorism. New York: Knopf, 1974.

South, S.; Doss, B. y Christensen, A. "Through the eyes of the beholder: the mediating role of relationship acceptance in the impact of partner behavior". Family Relations 59. 2010: 611-622.

Fecha de ingreso: 16/11/2011 Fecha de aprobación: 17/08/2012 
